\title{
The Schwinger SU(3) construction. I. Multiplicity problem and relation to induced representations
}

\author{
S. Chaturvedia) \\ School of Physics, University of Hyderabad, Hyderabad 500046, India \\ N. Mukundab) \\ Centre for Theoretical Studies, Indian Institute of Science, Bangalore 560012, India
}

\begin{abstract}
The Schwinger oscillator operator representation of SU(3) is analyzed with particular reference to the problem of multiplicity of irreducible representations. It is shown that with the use of an $\operatorname{Sp}(2, R)$ unitary representation commuting with the $\mathrm{SU}(3)$ representation, the infinity of occurrences of each $\mathrm{SU}(3)$ irreducible representation can be handled in complete detail. A natural "generating representation" for $\mathrm{SU}(3)$, containing each irreducible representation exactly once, is identified within a subspace of the Schwinger construction, and this is shown to be equivalent to an induced representation of $\mathrm{SU}(3)$.
\end{abstract}

\section{INTRODUCTION}

The well known Schwinger representation of the Lie algebra of SU(2), ${ }^{1}$ constructed using the annihilation and creation operators of two independent quantum mechanical harmonic oscillators, has played an important role in many widely differing contexts. Within the quantum theory of angular momentum it has made the calculation of various quantities somewhat easier than by other methods. Beyond this, it has been very effectively exploited in the physics of strongly correlated systems, ${ }^{2}$ in quantum optics of two mode radiation fields, ${ }^{3}$ and in the study of certain classes of partially coherent optical beams, ${ }^{4}$ namely to obtain the coherent mode decomposition of anisotropic Gaussian Schell model beams. It arises quite naturally in the context of a classical description of particles with non-Abelian charges ${ }^{5}$ and has also been used in a recent investigation of the Pauli spin-statistics theorem. ${ }^{6}$

Bargmann has presented an entire function Hilbert space analog of the Schwinger construction, which is extremely elegant and possesses special merits of its own. ${ }^{7}$ This may be viewed as a counterpart to the Fock space description of quantum mechanical oscillator systems.

Certain specially attractive features of the Schwinger SU(2) construction should be mentioned. It leads upon exponentiation to a unitary representation (UR) of SU(2) in which each unitary irreducible representation (UIR), labeled as usual by the spin quantum number $j$ with possible values $0, \frac{1}{2}, 1, \ldots$, appears exactly once. In other words, it is complete in the sense that no UIR of $\mathrm{SU}(2)$ is missed, and also economical in the sense of being multiplicity free. Thus, reflecting these two features, it may be regarded as a "generating representation" for SU(2), a concept that has been effectively used in understanding the structures of various kinds of ClebschGordan series for UIRs of the noncompact group SU $(1,1) .{ }^{8}$ In addition, of course, the use of boson operator methods makes many operator and state vector calculations relatively easy to carry out.

It is of considerable interest to extend the Schwinger construction to other compact Lie groups, the next natural case after $\mathrm{SU}(2)$ being $\mathrm{SU}(3)$. The aims behind any such attempt would be

\footnotetext{
${ }^{a)}$ Electronic mail: scsp@uohyd.ernet.in

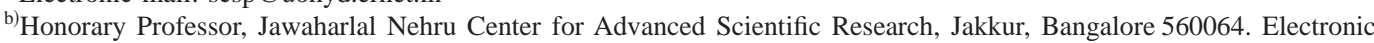
mail:nmukunda@cts.iisc.ernet.in
} 
to preserve the simplicity of the boson calculus, to cover all UIRs of the concerned group, and to do it in a multiplicity free manner.

The case of SU(3) has been studied by several authors since the work of Moshinsky. ${ }^{9}$ The aim of the present article is somewhat different from previous studies, being motivated by the particular points of view mentioned above. In particular our aim is to see to what extent the attractive features of the $\mathrm{SU}(2)$ construction survive when we consider $\mathrm{SU}(3)$, and which ones have to be given up.

A brief overview of this article is as follows. In Sec. II we collect together some relevant facts regarding unitary representations of compact Lie groups with special attention to SU(3). In particular, we highlight the fact that the theory of induced representations leads to a unitary representation of SU(3) which has all the properties becoming of a "generating representation" of SU(3) in that it contains all the UIRs of SU(3) exactly once each. The Hilbert space carrying this unitary representation turns out to be the Hilbert space of functions on unit sphere in $C^{3}$. In Sec. III, we turn to the Schwinger oscillator construction for SU(3) and show that a naive extension of the Schwinger SU(2)-construction making use of six oscillators leads to a very "fat" UR of SU(3) containing each UIR of $\mathrm{SU}(3)$ infinitely many times. We then show how the group $\operatorname{Sp}(2, R)$ enables us to completely handle this multiplicity and also neatly isolate from this rather large space a subspace carrying a UR of SU(3) of a "generating representation" type. At this stage, we have two "generating representations" of SU(3), one based on the Hilbert space of functions on a unit sphere in $C^{3}$ and the other based on the Fock space of six oscillators, and a natural question to ask is how the two are related. To this end, in Sec. IV, we make use of the Bargmann representation, to transcribe the Fock space description into a description based on a Hilbert space of square integrable functions in six complex variables satisfying certain conditions. This transcription enables us to establish an equivalence map between the Hilbert spaces supporting the two incarnations of the "generating representation" for SU(3), details of which are given in Secs. V and VI. Section VII contains concluding remarks and further outlook, and an appendix gives the details of the construction of $\mathrm{SU}(3) \times \mathrm{Sp}(2, R)$ basis states.

\section{UNITARY REPRESENTATIONS OF COMPACT LIE GROUPS, THE SU(3) CASE}

It is useful to first recall some basic facts concerning the representation theory of any compact simple Lie group $G$. The basic building blocks are the UIRs of $G$. Each UIR carries certain identifying labels (eigenvalues of Casimir operators), such as $j$ for $\mathrm{SU}(2)$. It is of a characteristic dimension, such as $2 j+1$ for $\mathrm{SU}(2)$. In addition, we may set up some convenient orthonormal basis in the space of the UIR, as simultaneous eigenvectors of some complete commuting set of Hermitian operators. The eigenvalue sets labeling the basis vectors are generalizations of the single magnetic quantum number $m$ for $\mathrm{SU}(2)$.

A general UR of $G$ is reducible into UIRs, each occurring with some multiplicity. Thus the UR as a whole is in principle completely determined upto equivalence by these multiplicities. However, certain URs have special significance, reflecting the way they are constructed, and so deserve special attention. We consider two cases - the regular representation, and representations induced from various Lie subgroups of $G$.

The Hilbert space carrying the regular representation of $G$ is the space $L^{2}(G)$ of all complex square integrable functions on $G$, the integration being with respect to the (left and right) translation invariant volume element on $G$. On this space there are in fact two (mutually commuting) regular representations of $G$, the left and the right regular representations. Upon reduction into UIRs each of these contains every UIR of $G$ without exception, the multiplicity of occurrence of a particular UIR is just its dimension. Thus the regular representations possess the completeness property of the Schwinger SU(2) construction, but not its economy.

Next we look at the family of induced URs of $G .{ }^{10}$ Let $H$ be some Lie subgroup of $G$, and let $D(h), h \in H$, be the operators of a UIR of $H$ on some Hilbert space $\mathcal{V}$. Then a certain unique UR of $G$, with operators $\mathcal{D}_{H}^{(\text {ind, } D)}(g)$ for $g \in G$, can be constructed. As the labels indicate, this UR is induced from the UIR $D(\cdot)$ of $H$. The Hilbert space $\mathcal{H}_{H}^{(\text {ind, } D)}$ of this UR consists of functions on 
$G$ with values in $\mathcal{V}$ obeying a covariance condition and having finite norm:

$$
\begin{gathered}
\psi \in \mathcal{H}_{H}^{(\mathrm{ind}, D)}: \quad \psi(g) \in \mathcal{V}, g \in G, \\
\psi(g h)=D\left(h^{-1}\right) \psi(g), h \in H, \\
\|\psi\|^{2}=\int_{G} d g(\psi(g), \psi(g))_{\mathcal{V}}<\infty .
\end{gathered}
$$

Here $d g$ is the (suitably normalized) invariant volume element on $G$, and the integrand is the squared norm of $\psi(g) \in \mathcal{V}$. The covariance condition means that $\psi(g)$ is essentially a function on the coset space $G / H$, in the sense that the "values" of $\psi(g)$ all over a coset are determined by its "value" at any one representative point. Correspondingly, due to unitarity of $D(h),(\psi(g), \psi(g))_{\mathcal{V}}$ is constant over each coset; so, the expression for $\|\psi\|^{2}$ can be simplified and expressed in terms of a $G$-invariant volume element on $G / H$. The action of $\mathcal{D}_{H}^{(\text {ind, }, D)}(g)$ on $\psi$ is then given by

$$
\begin{gathered}
g \in G: \mathcal{D}_{H}^{(\text {ind, } D)}(g) \psi=\psi^{\prime}, \\
\psi^{\prime}\left(g^{\prime}\right)=\psi\left(g^{-1} g^{\prime}\right) .
\end{gathered}
$$

It is clear that $G$ action preserves the covariance condition, and we have a UR of $G$ on $\mathcal{H}_{H}^{(\text {ind, } D)}$.

Whereas $D(\cdot)$ was assumed to be a UIR of $H, \mathcal{D}_{H}^{(\text {ind,D) }}(\cdot)$ is in general reducible; so it is a direct sum of the various UIRs of $G$, each occurring with some multiplicity. These multiplicities are determined by the reciprocity theorem: ${ }^{10}$ Each UIR $\mathcal{D}(\cdot)$ of $G$ appears in $\mathcal{D}_{H}^{(\text {ind, }, D)}(\cdot)$ as often as $\mathcal{D}(\cdot)$ contains $D(\cdot)$ upon restriction from $G$ to $H$.

With this general background we now take up the specific case of SU(3). The defining representation of this group is

$$
\mathrm{SU}(3)=\left\{A=3 \times 3 \text { complex matrix } \mid A^{\dagger} A=I_{3 \times 3}, \operatorname{det} A=1\right\},
$$

with the group operation given by matrix multiplication. In this representation the eight Hermitian generators are $\frac{1}{2} \lambda_{\alpha}, \alpha=1,2, \ldots, 8$, where the matrices $\lambda_{\alpha}$ and the structure constants $f_{\alpha \beta \gamma}$ occurring in the commutation relations

$$
\left[\lambda_{\alpha}, \lambda_{\beta}\right]=2 i f_{\alpha \beta \gamma} \lambda_{\gamma}, \quad \alpha, \beta, \gamma=1,2, \ldots, 8,
$$

are all very well known. ${ }^{11}$

A general UIR of SU(3) is determined by two independent nonnegative integers $p$ and $q$, so it may be denoted as $(p, q)$. It is of dimension $d(p, q)=\frac{1}{2}(p+1)(q+1)(p+q+2)$. The defining three-dimensional UIR in (2.3) is $(1,0)$, while the inequivalent complex conjugate UIR is $(0,1)$. In general the complex conjugate of $(p, q)$ is $(q, p)$, and the adjoint UIR is $(1,1)$ of dimension eight. Various choices of "magnetic quantum numbers" within a UIR may be made. The one corresponding to the canonical subgroup $\mathrm{SU}(2) \times \mathrm{U}(1) / Z_{2}=\mathrm{U}(2) \subset \mathrm{SU}(3)$ leads to the three quantum numbers $I, M, Y$ in standard notation. Here $I$ and $M$ are the isospin and magnetic quantum number labels for a general UIR of SU(2), while $Y$ is the eigenvalue of the (suitably normalized) $\mathrm{U}(1)$ or hypercharge generator. The subgroups $\mathrm{SU}(2)$ and $\mathrm{U}(1)$ commute, and for definiteness we take SU(2) to be the one acting on the first two dimensions of the three dimensions in the UIR $(1,0)$. The spectrum of " $I-Y$ " multiplets present in the UIR $(p, q)$ can be described thus:

$$
I=\frac{1}{2}(r+s), \quad Y=r-s+\frac{2}{3}(q-p), \quad 0 \leqslant r \leqslant p, \quad 0 \leqslant s \leqslant q .
$$

Thus for each pair of integers $(r, s)$ in the above ranges, we have one $I-Y$ multiplet, with $M$ going over the usual $2 I+1$ values $I, I-1, \ldots,-I+1,-I$. Then the orthonormal basis vectors for the UIR $(p, q)$ of $\mathrm{SU}(3)$ may be written as $|p, q ; I M Y\rangle$. This UIR can be realized via suitably 
constructed irreducible tensors. A tensor $T$ with $p$ indices belonging to the UIR $(1,0)$ and $q$ indices to the UIR $(0,1)$ is a collection of complex components $T_{k_{1} \cdots k_{q}}^{j_{1} \cdots j_{p}}, j$ and $k=1,2,3$, transforming under $A \in \mathrm{SU}(3)$ by the rule

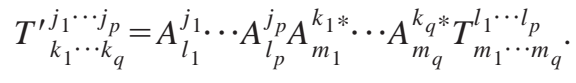

If in addition $T$ is completely symmetric separately in the superscripts and in the subscripts, and is traceless, i.e., contraction of any upper index with any lower index leads to zero, then all these properties are maintained under $\mathrm{SU}(3)$ action and $T$ is an irreducible tensor. It then has precisely $d(p, q)$ independent components (in the complex sense), and the space of all such tensors carries the UIR $(p, q)$. The explicit transition from the tensor components $T_{k_{1} \cdots k_{q}}^{j_{1} \cdots j_{p}}$ to the canonical components $T_{I M Y}^{(p, q)}$ may be found in Ref. 12

The regular representations of $\mathrm{SU}(3)$ act on the space $L^{2}(\mathrm{SU}(3))$, and in each of them the UIR $(p, q)$ appears $d(p, q)$ times. We shall not be concerned with this UR of SU(3) in our work. Instead we give now the UIR contents of some selected induced URs of SU(3). For illustrative purposes we consider the following four subgroups:

$$
\begin{gathered}
\mathrm{U}(1) \times \mathrm{U}(1)=\left\{A=\operatorname{diag}\left(e^{i\left(\theta_{1}+\theta_{2}\right)}, e^{i\left(\theta_{1}-\theta_{2}\right)}, e^{-2 i \theta_{1}}\right) \mid 0 \leqslant \theta_{1}, \theta_{2} \leqslant 2 \pi\right\} ; \\
\mathrm{SU}(2)=\left\{A=\left(\begin{array}{ll}
a & \mathbf{0} \\
\mathbf{0} & 1
\end{array}\right) \mid a \in \mathrm{SU}(2)\right\} ; \\
\mathrm{U}(2)=\left\{A=\left(\begin{array}{cc}
u & \mathbf{0} \\
\mathbf{0} & (\operatorname{det} u)^{-1}
\end{array}\right) \mid u \in \mathrm{U}(2)\right\} ; \\
\mathrm{SO}(3)=\left\{A \in \mathrm{SU}(3) \mid A^{*}=A\right\} .
\end{gathered}
$$

In each case, we look at the induced UR of SU(3) arising from the trivial one-dimensional UIR of the subgroup. In the first two cases, in order to apply the reciprocity theorem, we can use the information in (2.5) giving the $\mathrm{SU}(2) \times \mathrm{U}(1) / Z_{2}$ content of the UIR $(p, q)$ of $\mathrm{SU}(3)$. Defining by a zero in the superscript the trivial UIR of the relevant subgroup, we have the results:

$$
\begin{gathered}
\mathcal{D}_{\mathrm{U}(1) \times \mathrm{U}(1)}^{(\mathrm{ind}, 0)}=\sum_{\substack{p, q=0,1, \cdots \\
p=q \bmod 3}}^{\infty} \oplus n_{p, q}(p, q), n_{p, q}=\min (p+1, q+1) ; \\
\mathcal{D}_{\mathrm{SU}(2)}^{(\mathrm{ind}, 0)}=\sum_{p, q=0,1, \cdots}^{\infty} \oplus(p, q) ; \\
\mathcal{D}_{\mathrm{U}(2)}^{(\text {ind, } 0)}=\sum_{p=0,1, \cdots}^{\infty} \oplus(p, p) .
\end{gathered}
$$

The real dimensions of the corresponding coset spaces $\mathrm{SU}(3) / \mathrm{U}(1) \times \mathrm{U}(1), \mathrm{SU}(3) / \mathrm{SU}(2)$ and $\mathrm{SU}(3) / \mathrm{U}(2)$ are 6,5 and 4 , respectively. In the case of induction from the trivial UIR of $\mathrm{SO}(3)$, we need to use the fact that the UIR $(p, q)$ of $\mathrm{SU}(3)$ does not contain an $\mathrm{SO}(3)$ invariant state if either $p$ or $q$ or both are odd, while it contains one such state if both $p$ and $q$ are even. Then we arrive at the reduction

$$
\mathcal{D}_{\mathrm{SO}(3)}^{(\text {ind }, 0)}=\sum_{r, s=0,1, \cdots}^{\infty} \oplus(2 r, 2 s)
$$

with $\mathrm{SU}(3) / \mathrm{SO}(3)$ being of real dimension 5. 
From the above discussion we see that the induced UR $\mathcal{D}_{\mathrm{SU}(2)}^{(\mathrm{ind}, 0)}$ of $\mathrm{SU}(3)$ is particularly interesting in that it captures both the completeness and the economy properties of the Schwinger $\mathrm{SU}(2)$ construction: each UIR of SU(3) is present, exactly once. Thus we may call this a generating representation of $\mathrm{SU}(3)$; it is much leaner than the regular representations.

\section{THE MINIMAL SU(3) SCHWINGER OSCILLATOR CONSTRUCTION}

An elementary oscillator operator construction of the SU(3) generators is based on three independent pairs of annihilation and creation operators $\hat{a}_{j}, \hat{a}_{j}^{\dagger}$ obeying

$$
\left[\hat{a}_{j}, \hat{a}_{k}^{\dagger}\right]=\delta_{j k}, \quad\left[\hat{a}_{j}, \hat{a}_{k}\right]=\left[\hat{a}_{j}^{\dagger}, \hat{a}_{k}^{\dagger}\right]=0, \quad j, k=1,2,3 .
$$

We write $\mathcal{H}^{(a)}$ for the Hilbert space on which these operators act irreducibly. The individual and total number operators are

$$
\hat{N}_{1}^{(a)}=\hat{a}_{1}^{\dagger} \hat{a}_{1}, \quad \hat{N}_{2}^{(a)}=\hat{a}_{2}^{\dagger} \hat{a}_{2}, \quad \hat{N}_{3}^{(a)}=\hat{a}_{3}^{\dagger} \hat{a}_{3}, \quad \hat{N}^{(a)}=\hat{a}_{j}^{\dagger} \hat{a}_{j} .
$$

If we now define the bilinear operators

$$
Q_{\alpha}^{(a)}=\frac{1}{2} \hat{a}^{\dagger} \lambda_{\alpha} \hat{a}, \quad \alpha=1,2, \ldots, 8,
$$

each $Q_{\alpha}^{(a)}$ is Hermitian, and they obey the SU(3) Lie algebra commutation relations

$$
\left[Q_{\alpha}^{(a)}, Q_{\beta}^{(a)}\right]=i f_{\alpha \beta \gamma} Q_{\gamma}^{(a)} \text {. }
$$

In addition they conserve the total number operator:

$$
\left[Q_{\alpha}^{(a)}, \hat{N}^{(a)}\right]=0
$$

Upon exponentiation of these generators we obtain a particular UR, $\mathcal{U}^{(a)}(A)$ say, of $\operatorname{SU}(3)$ acting on $\mathcal{H}^{(a)}$, under which the creation (annihilation) operators $\hat{a}_{j}^{\dagger}\left(\hat{a}_{j}\right)$ transform via the UIR $(1,0)$ $((0,1))$ :

$$
\begin{aligned}
& \mathcal{U}^{(a)}(A) \hat{a}_{j}^{\dagger} \mathcal{U}^{(a)}(A)^{-1}=A_{j}^{k} \hat{a}_{k}^{\dagger}, \\
& \mathcal{U}^{(a)}(A) \hat{a}_{j} \mathcal{U}^{(a)}(A)^{-1}=A_{j}^{k^{*}} \hat{a}_{k} .
\end{aligned}
$$

However, upon reduction, $\mathcal{U}^{(a)}(A)$ contains only the "triangular" UIRs $(p, 0)$ of SU(3), once each. In that sense this UR may be regarded as the "generating representation" for this subset of UIRs. For any given $p \geqslant 0$, the $\operatorname{UIR}(p, 0)$ is realized on that subspace $\mathcal{H}^{(p, 0)}$ of $\mathcal{H}^{(a)}$ over which the total number operator $\hat{N}^{(a)}$ takes the eigenvalue $p$; and the connection between the tensor and the Fock space descriptions is given in this manner:

$$
\begin{aligned}
& \left\{T^{\left.j_{1} \cdots j_{p}\right\}} \rightarrow|T\rangle=T^{j_{1} \cdots j_{p}} \hat{a}_{j_{1}}^{\dagger} \cdots \hat{a}_{j_{p}}^{\dagger}|\underline{0}\rangle \in \mathcal{H}^{(p, 0)} \subset \mathcal{H}^{(a)},\right. \\
& \hat{a}_{j}|\underline{0}\rangle=0 \\
& \mathcal{U}^{(a)}(A)|T\rangle=\left|T^{\prime}\right\rangle,
\end{aligned}
$$

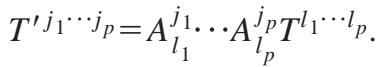

Therefore we have the (orthogonal) direct sum decompositions 


$$
\begin{aligned}
\mathcal{H}^{(a)} & =\sum_{p=0,1, \cdots}^{\infty} \oplus \mathcal{H}^{(p, 0)}, \\
\mathcal{H}^{(p, 0)} & =\operatorname{Sp}\left\{\hat{a}_{j_{1}}^{\dagger} \cdots \hat{a}_{j_{p}}^{\dagger}|\underline{0}\rangle\right\}, \\
\mathcal{U}^{(a)} & =\sum_{p=0,1, \cdots}^{\infty} \oplus(p, 0) .
\end{aligned}
$$

To be able to obtain the other UIRs as well, we bring in another independent triplet of oscillator operators $\hat{b}_{j}$ and $\hat{b}_{j}^{\dagger}$ obeying the same commutation relations (3.1) and commuting with $\hat{a}$ 's and $\hat{a}^{\dagger}$ 's:

$$
\begin{gathered}
{\left[\hat{b}_{j}, \hat{b}_{k}^{\dagger}\right]=\delta_{j k}, \quad\left[\hat{b}_{j}, \hat{b}_{k}\right]=\left[\hat{b}_{j}^{\dagger}, \hat{b}_{k}^{\dagger}\right]=0, \quad j, k=1,2,3,} \\
{\left[\hat{a}_{j} \text { or } \hat{a}_{j}^{\dagger}, \hat{b}_{k} \text { or } \hat{b}_{k}^{\dagger}\right]=0 .}
\end{gathered}
$$

The corresponding Hilbert space is $\mathcal{H}^{(b)}$, and the $b$-type number operators are

$$
\hat{N}_{1}^{(b)}=\hat{b}_{1}^{\dagger} \hat{b}_{1}, \quad \hat{N}_{2}^{(b)}=\hat{b}_{2}^{\dagger} \hat{b}_{2}, \quad \hat{N}_{3}^{(b)}=\hat{b}_{3}^{\dagger} \hat{b}_{3}, \quad \hat{N}^{(b)}=\hat{b}_{j}^{\dagger} \hat{b}_{j} .
$$

We define the $b$-type $\mathrm{SU}(3)$ generators as

$$
Q_{\alpha}^{(b)}=-\frac{1}{2} \hat{b}^{\dagger} \lambda_{\alpha}^{*} \hat{b}, \quad \alpha=1,2, \ldots, 8,
$$

and they obey

$$
\begin{gathered}
{\left[Q_{\alpha}^{(b)}, Q_{\beta}^{(b)}\right]=i f_{\alpha \beta \gamma} Q_{\gamma}^{(b)},} \\
{\left[Q_{\alpha}^{(b)}, \hat{N}^{(b)}\right]=0 .}
\end{gathered}
$$

Exponentiation of these generators leads to a UR $\mathcal{U}^{(b)}(A)$ acting on $\mathcal{H}^{(b)}$, under which the creation (annihilation) operators $\hat{b}_{j}^{\dagger}\left(\hat{b}_{j}\right)$ transform via the UIR $(0,1)((1,0))$ :

$$
\begin{gathered}
\mathcal{U}^{(b)}(A) \hat{b}_{j}^{\dagger} \mathcal{U}^{(b)}(A)^{-1}=A_{j}^{k^{*}} \hat{b}_{k}^{\dagger}, \\
\mathcal{U}^{(b)}(A) \hat{b}_{j} \mathcal{U}^{(b)}(A)^{-1}=A_{j}^{k} \hat{b}_{k}
\end{gathered}
$$

Now this UR of SU(3) contains each of the triangular UIRs $(0, q)$ for $q \geqslant 0$ once each, so it is a generating representation for this family of UIRs. For each $q \geqslant 0$, the UIR $(0, q)$ is realized on that subspace $\mathcal{H}^{(0, q)}$ of $\mathcal{H}^{(b)}$ over which the total number operator $\hat{N}^{(b)}$ takes the eigenvalue $q$. Analogous to (3.7), the tensor-Fock space connection is now

$$
\begin{gathered}
\left\{T_{k_{1} \cdots k_{q}}\right\} \rightarrow|T\rangle=T_{k_{1} \cdots k_{q}} \hat{b}_{k_{1}}^{\dagger} \cdots \hat{b}_{k_{q}}^{\dagger}|\underline{0}\rangle \in \mathcal{H}^{(0, q)} \subset \mathcal{H}^{(b)}, \\
\hat{b}_{k}|\underline{\underline{0}}\rangle=0 \\
\mathcal{U}^{(b)}(A)|T\rangle=\left|T^{\prime}\right\rangle \\
T^{\prime}{ }_{k_{1} \cdots k_{q}}=A_{m_{1}}^{k_{1} *} \cdots A_{m_{q}}^{k_{q^{*}}} T_{m_{1} \cdots m_{q}} .
\end{gathered}
$$


[The use of a common symbol $|\underline{\underline{0}}\rangle$ for the Fock ground states in $\mathcal{H}^{(a)}$ and $\mathcal{H}^{(b)}$, and $|T\rangle$ in (3.7) and (3.14), should cause no confusion as the meanings are always clear from the context.] In place of (3.8) we now have

$$
\begin{aligned}
\mathcal{H}^{(b)} & =\sum_{q=0,1, \cdots}^{\infty} \oplus \mathcal{H}^{(0, q)}, \\
\mathcal{H}^{(0, q)} & =\operatorname{Sp}\left\{\hat{b}_{k_{1}}^{\dagger} \cdots \hat{b}_{k_{q}}^{\dagger}|\underline{0}\rangle\right\}, \\
\mathcal{U}^{(b)} & =\sum_{q=0,1, \cdots}^{\infty} \oplus(0, q) .
\end{aligned}
$$

From these considerations it is clear that if we want to obtain all the UIRs $(p, q)$ of $\operatorname{SU}(3)$, missing none, the minimal scheme is to use all six independent oscillators $\hat{a}_{j}, \hat{a}_{j}^{\dagger}, \hat{b}_{j}, \hat{b}_{j}^{\dagger}$ and define the $\mathrm{SU}(3)$ generators $^{13}$

$$
Q_{\alpha}=Q_{\alpha}^{(a)}+Q_{\alpha}^{(b)}
$$

They act on the product Hilbert space $\mathcal{H}=\mathcal{H}^{(a)} \times \mathcal{H}^{(b)}$, of course obey the SU(3) commutation relations, and upon exponentiation lead to the $\operatorname{UR} \mathcal{U}(A)=\mathcal{U}^{(a)}(A) \times \mathcal{U}^{(b)}(A)$. However, as we see in a moment, while each $\operatorname{UIR}(p, q)$ is certainly present in $\mathcal{U}(A)$, it occurs infinitely many times. A systematic group theoretic procedure to handle this multiplicity, based on the noncompact group $\operatorname{Sp}(2, R)$, will be set up below. The tensor-Fock space connection is now given as follows. To an irreducible tensor $T_{k_{1} \cdots k_{q}}^{j_{1} \cdots j_{p}}$ which is symmetric and traceless and so "belongs" to the UIR $(p, q)$ we associate the vector $|T\rangle \in \mathcal{H}$ by

$$
\begin{gathered}
|T\rangle=T_{k_{1} \cdots k_{q}}^{j_{1} \cdots j_{p}} \hat{a}_{j_{1}}^{\dagger} \cdots \hat{a}_{j_{p}}^{\dagger} \hat{b}_{k_{1}}^{\dagger} \cdots \hat{b}_{k_{q}}^{\dagger}|\underline{0}, \underline{0}\rangle \in \mathcal{H}^{(p, 0)} \times \mathcal{H}^{(0, q)} \subset \mathcal{H}, \\
\hat{a}_{j}|\underline{0}, \underline{0}\rangle=\hat{b}_{j}|\underline{0}, \underline{0}\rangle=0, \\
\mathcal{U}(A)|T\rangle=\left|T^{\prime}\right\rangle,
\end{gathered}
$$

the components of $T^{\prime}$ being given by (2.6). While this vector $|T\rangle$ is certainly a simultaneous eigenvector of the two number operators $\hat{N}^{(a)}, \hat{N}^{(b)}$ with eigenvalues $p, q$, respectively, the tracelessness of the tensor $T_{k_{1} \cdots k_{q}}^{j_{1} \cdots j_{p}}$ implies that (unless at least one of $p$ and $q$ vanishes) we do not get all such independent vectors in $\mathcal{H}$. This aspect is further clarified below. On the other hand, if we drop the tracelessness condition and retain only symmetry, we do span all of $\mathcal{H}^{(p, 0)} \times \mathcal{H}^{(0, q)}$ via (3.17).

The decomposition of $\mathcal{U}(A)$ into UIRs, and the counting of multiplicities, is accomplished by appealing to the Clebsch-Gordan series for the product of two triangular UIRs $(p, 0)$ and $(0, q):{ }^{14}$

$$
(p, 0) \times(0, q)=(p, q) \oplus(p-1, q-1) \oplus(p-2, q-2) \oplus \ldots \oplus(p-r, q-r), \quad r=\min (p, q) .
$$

Therefore, at the Hilbert space level one has the orthogonal subspace decomposition

$$
\begin{gathered}
\mathcal{H}=\mathcal{H}^{(a)} \times \mathcal{H}^{(b)}=\left(\sum_{p=0,1, \cdots}^{\infty} \oplus \mathcal{H}^{(p, 0)}\right) \times\left(\sum_{q=0,1, \cdots}^{\infty} \oplus \mathcal{H}^{(0, q)}\right)=\sum_{p, q=0,1, \cdots}^{\infty} \oplus \mathcal{H}^{(p, 0)} \times \mathcal{H}^{(0, q)}, \\
\mathcal{H}^{(p, 0)} \times \mathcal{H}^{(0, q)}=\sum_{\rho=0,1, \cdots}^{r} \oplus \mathcal{H}^{(p-\rho, q-\rho ; \rho)}, \quad r=\min (p, q) .
\end{gathered}
$$


Here $\mathcal{H}^{(p-\rho, q-\rho ; \rho)}$ is that unique subspace of $\mathcal{H}^{(p, 0)} \times \mathcal{H}^{(0, q)}$ carrying the UIR $(p-\rho, q-\rho)$ present on the right hand side of (3.18). All vectors in $\mathcal{H}^{(p-\rho, q-\rho ; \rho)}$ are eigenvectors of $\hat{N}^{(a)}$ and $\hat{N}^{(b)}$ with eigenvalues $p$ and $q$, respectively; and if the tensor $T$ in (3.17) is assumed traceless, only vectors in $\mathcal{H}^{(p, q ; 0)} \subset \mathcal{H}^{(p, 0)} \times \mathcal{H}^{(0, q)}$ are obtained on the right in that equation.

Focusing on a given UIR $(p, q)$, we see that it appears once each in $\mathcal{H}^{(p, 0)}$ $\times \mathcal{H}^{(0, q)}, \mathcal{H}^{(p+1,0)} \times \mathcal{H}^{(0, q+1)}, \ldots$, in the respective irreducible subspaces $\mathcal{H}^{(p, q ; 0)}, \mathcal{H}^{(p, q ; 1)}, \ldots$ Thus it is the leading piece in $\mathcal{H}^{(p, 0)} \times \mathcal{H}^{(0, q)}$, the next to the leading piece in $\mathcal{H}^{(p+1,0)}$ $\times \mathcal{H}^{(0, q+1)}$, and so on. Therefore, the decomposition (3.19) of $\mathcal{H}$ can be presented in the alternative manner

$$
\mathcal{H}=\sum_{p, q=0,1 \ldots}^{\infty} \oplus \sum_{\rho=0,1 \ldots}^{\infty} \oplus \mathcal{H}^{(p, q ; \rho)}, \mathcal{H}^{(p, q ; \rho)} \subset \mathcal{H}^{(p+\rho, 0)} \times \mathcal{H}^{(0, q+\rho)},
$$

each $\mathcal{H}^{(p, q ; \rho)}$ carrying the same UIR $(p, q)$. Thus the index $\rho$ is an (orthogonal) multiplicity label with an infinite number of values. For $\rho \neq \rho^{\prime}, \mathcal{H}^{\left(p, q ; \rho^{\prime}\right)}$ and $\mathcal{H}^{(p, q ; \rho)}$ are mutually orthogonal. This is also evident as $\hat{N}^{(a)}=p+\rho^{\prime}, \hat{N}^{(b)}=q+\rho^{\prime}$ in the former and $\hat{N}^{(a)}=p+\rho, \hat{N}^{(b)}=q+\rho$ in the latter.

We now introduce the group $\operatorname{Sp}(2, R)$ to handle in a systematic way the multiplicity index $\rho$. The Hermitian generators of $\operatorname{Sp}(2, R)$ and their commutation relations are ${ }^{15}$

$$
\begin{gathered}
J_{0}=\frac{1}{2}\left(\hat{N}^{(a)}+\hat{N}^{(b)}+3\right), \\
K_{1}=\frac{1}{2}\left(\hat{a}_{j}^{\dagger} \hat{b}_{j}^{\dagger}+\hat{a}_{j} \hat{b}_{j}\right), \\
K_{2}=-\frac{i}{2}\left(\hat{a}_{j}^{\dagger} \hat{b}_{j}^{\dagger}-\hat{a}_{j} \hat{b}_{j}\right) ; \\
{\left[J_{0}, K_{1}\right]=i K_{2}, \quad\left[J_{0}, K_{2}\right]=-i K_{1}, \quad\left[K_{1}, K_{2}\right]=-i J_{0} .}
\end{gathered}
$$

Using the raising and lowering combinations $K_{ \pm}=K_{1} \pm i K_{2}$ we have

$$
\begin{gathered}
K_{+}=\hat{a}_{j}^{\dagger} \hat{b}_{j}^{\dagger}, \quad K_{-}=K_{+}^{\dagger}=\hat{a}_{j} \hat{b}_{j} ; \\
{\left[J_{0}, K_{ \pm}\right]= \pm K_{ \pm}, \quad\left[K_{+}, K_{-}\right]=-2 J_{0} .}
\end{gathered}
$$

The significance of this construction is that the two groups $\mathrm{SU}(3)$ and $\mathrm{Sp}(2, R)$, both acting unitarily on $\mathcal{H}$, commute with one another:

$$
\left[J_{0} \text { or } K_{1} \text { or } K_{2}, Q_{\alpha}\right]=0 \text {. }
$$

It is this that helps us handle the multiplicity of occurrences of each $\operatorname{SU}(3)$ UIR $(p, q)$ in $\mathcal{H}: \rho$ becoming a "magnetic quantum number" within a suitable UIR of $\operatorname{Sp}(2, R)$.

The family of (infinite dimensional) UIRs of $\operatorname{Sp}(2, R)$ relevant here is the positive discrete family $D_{k}^{(+)}$, labeled by $k=\frac{1}{2}, 1, \frac{3}{2}, 2,2 \ldots$ (Actually we encounter only $k \geqslant \frac{3}{2}$.) Within the UIR $D_{k}^{(+)}$ we have an orthonormal basis $|k, m\rangle$ on which the generators act as follows: ${ }^{16}$

$$
\begin{aligned}
& J_{0}|k, m\rangle=m|k, m\rangle, \quad m=k, k+1, k+2, \ldots, \\
& K_{ \pm}|k, m\rangle=\sqrt{(m \pm k)(m \mp k \pm 1)}|k, m \pm 1\rangle .
\end{aligned}
$$

From these follow the useful results

$$
K_{1}^{2}+K_{2}^{2}-J_{0}^{2}=k(1-k),
$$




$$
\begin{gathered}
|k, m\rangle=\sqrt{\frac{(2 k-1) !}{(m-k) !(m+k-1) !}} K_{+}^{m-k}|k, k\rangle, \\
K_{+}^{m-k} K_{-}^{m-k}|k, m\rangle=\frac{(m-k) !(m+k-1) !}{(2 k-1) !}|k, m\rangle .
\end{gathered}
$$

Going back to the generators (3.21) it is clear that on all of $\mathcal{H}^{(p, 0)} \times \mathcal{H}^{(0, q)}$, and so on each $\mathcal{H}^{(p-\rho, q-\rho ; \rho)}, J_{0}$ has the eigenvalue $\frac{1}{2}(p+q+3)$; therefore on $\mathcal{H}^{(p, q ; \rho)}$ it has the eigenvalue $\frac{1}{2}(p+q+3)+\rho$. It is also clear that action by $K_{ \pm}$on $\mathcal{H}^{(p, 0)} \times \mathcal{H}^{(0, q)}$ leads to a subspace of $\mathcal{H}^{(p \pm 1,0)} \times \mathcal{H}^{(0, q \pm 1)}$. Therefore, because of $(3.23)$, we see that $K_{ \pm}$acting on $\mathcal{H}^{(p, q ; \rho)}$ yield $\mathcal{H}^{(p, q ; \rho \pm 1)}$. Of course $\mathcal{H}^{(p, q ; 0)}$ is annihilated by $K_{-}$.

Reflecting all this we see that an orthonormal basis for $\mathcal{H}$ can be set up labeled as follows:

$$
\begin{gathered}
|p, q ; I M Y ; m\rangle: p, q=0,1,2, \ldots ; \\
m=k, k+1, k+2, \ldots, \\
k=\frac{1}{2}(p+q+3) ; \\
N^{(a)}=p+m-k, \quad N^{(b)}=q+m-k .
\end{gathered}
$$

Since $k$ is determined in terms of $p$ and $q$, we do not include it as an additional label in the basis kets above. [The ranges for $I, M, Y$ within the $\mathrm{SU}(3) \mathrm{UIR}(p, q)$ are given in (2.5).] The $\mathrm{SU}(3)$ UIR labels $p, q$ determine $k$ and so the associated UIR $D_{k}^{(+)}$of $\operatorname{Sp}(2, R)$. For fixed $p, q$ as $I, M$, $Y, m$ vary we get a set of states carrying the $\operatorname{UIR}(p, q) \times D_{k}^{(+)}$of $\operatorname{SU}(3) \times \operatorname{Sp}(2, R)$. We can now appreciate the following relationships:

$$
\begin{gathered}
\mathcal{H}^{(p, q ; \rho)}=\operatorname{Sp}\{|p, q ; I M Y ; k+\rho\rangle \mid I M Y \text { varying }\}, \\
\rho=0,1,2, \ldots ; \\
\mathcal{H}^{(p, q ; \rho)}=K_{+}^{\rho} \mathcal{H}^{(p, q ; 0)} \\
K_{-} \mathcal{H}^{(p, q ; 0)}=0 .
\end{gathered}
$$

Therefore, the null space of $K_{-}$within $\mathcal{H}$ is the subspace

$$
\mathcal{H}_{0}=\sum_{p, q=0,1, \ldots}^{\infty} \oplus \mathcal{H}^{(p, q ; 0)}=\operatorname{Sp}\{|p, q ; I M Y ; k\rangle \mid p, q, I M Y \text { varying }\},
$$

and we see that the UR $\mathcal{U}(A)$ of $\mathrm{SU}(3)$ on $\mathcal{H}$ when restricted to $\mathcal{H}_{0}$ gives a UR $\mathcal{D}_{0}$ which is multiplicity free and includes every UIR of SU(3). It is thus identical in structure to the induced representation $\mathcal{D}_{\mathrm{SU}(2)}^{(\text {ind,0) }}$ in $(2.8 \mathrm{~b})$. We see how the use of $\mathrm{Sp}(2, R)$ helps us isolate $\mathcal{H}_{0}$ in a neat manner.

In addition to the subspaces $\mathcal{H}^{(p, q ; \rho)}, \mathcal{H}_{0}$ of $\mathcal{H}$ defined above, it is also useful to define the series of mutually orthogonal infinite dimensional subspaces

$$
\begin{gathered}
\mathcal{H}^{(p, q)}=\sum_{\rho=0}^{\infty} \oplus \mathcal{H}^{(p, q ; \rho)}=\operatorname{Sp}\{|p, q ; I M Y ; m\rangle \mid I M Y m \text { varying }\}, \\
p, q=0,1,2, \ldots
\end{gathered}
$$

Thus the infinity of occurrences of the $\operatorname{SU}(3)$ UIR $(p, q)$ are collected together in $\mathcal{H}^{(p, q)}$. 
In the Appendix we give explicit formulas for the state vectors $|p, q ; I M Y ; m\rangle$ as functions of the operators $\hat{a}_{j}^{\dagger}, \hat{b}_{j}^{\dagger}$ acting on the Fock vacuum $|\underline{0}, \underline{0}\rangle$.

\section{THE BARGMANN REPRESENTATION}

For some purposes the use of the Bargmann representation of the canonical commutation relations is more convenient than the Fock space description. ${ }^{17}$ We outline the definitions of $\mathcal{H}$ and the $\mathrm{SU}(3) \mathrm{UR} \mathcal{U}(A)=\mathcal{U}^{(a)}(A) \times \mathcal{U}^{(b)}(A)$ in this language, and then turn to the problem of isolating the subspace $\mathcal{H}_{0}$ in $\mathcal{H}$.

Vectors in $\mathcal{H}$ correspond to entire functions $f(\underline{z}, \underline{w})$ in six independent complex variables $\underline{z}$ $=\left(z_{j}\right), \underline{w}=\left(w_{j}\right), j=1,2,3$, with the squared norm defined as

$$
\|f\|^{2}=\int \prod_{j=1}^{3}\left(\frac{d^{2} z_{j}}{\pi}\right)\left(\frac{d^{2} w_{j}}{\pi}\right) e^{-z^{\dagger} z-w^{\dagger} w}|f(\underline{z}, \underline{w})|^{2} .
$$

Any such $f(\underline{z}, \underline{w})$ has a unique Taylor series expansion

$$
f(\underline{z}, \underline{w})=\sum_{p, q=0,1, \cdots}^{\infty} f_{k_{1} \cdots k_{q}}^{j_{1} \cdots j_{p}} z_{j_{1}} \cdots z_{j_{p}} w_{k_{1}} \cdots w_{k_{q}},
$$

involving the tensor components $f_{k_{1} \cdots k_{q}}^{j_{1} \cdots j_{p}}$ separately symmetric in the superscripts and the subscripts. In terms of these the squared norm is

$$
\|f\|^{2}=\sum_{p, q=0,1, \cdots}^{\infty} p ! q ! f_{k_{1} \cdots k_{q}}^{j_{1} \cdots j_{p}}{ }^{*} f_{k_{1} \cdots k_{q}}^{j_{1} \cdots j_{p}}
$$

The operators $\hat{a}_{j}, \hat{a}_{j}^{\dagger}, \hat{b}_{j}, \hat{b}_{j}^{\dagger}$ act on $f(\underline{z}, \underline{w})$ as follows:

$$
\hat{a}_{j} \rightarrow \frac{\partial}{\partial z_{j}}, \quad \hat{a}_{j}^{\dagger} \rightarrow z_{j}, \quad \hat{b}_{j} \rightarrow \frac{\partial}{\partial w_{j}}, \quad \hat{b}_{j}^{\dagger} \rightarrow w_{j} .
$$

The UR $\mathcal{U}(A)$ of $\mathrm{SU}(3)$ acts very simply via point transformations:

$$
(\mathcal{U}(A) f)(\underline{z}, \underline{w})=f\left(A^{-1} \underline{z}, A^{-1 *} \underline{w}\right) .
$$

The $\operatorname{Sp}(2, R)$ generators are particularly simple:

$$
\begin{gathered}
J_{0}=\frac{1}{2}\left(z_{j} \frac{\partial}{\partial z_{j}}+w_{j} \frac{\partial}{\partial w_{j}}+3\right), \\
K_{+}=z_{j} w_{j} \equiv \underline{z} \cdot \underline{w}, \\
K_{-}=\frac{\partial^{2}}{\partial z_{j} \partial w_{j}} \equiv \frac{\partial}{\partial \underline{z}} \cdot \frac{\partial}{\partial \underline{w}} .
\end{gathered}
$$

We will use these below.

It is clear that the terms in (4.2) and (4.3) for fixed $p$ and $q$ are contributions from $\mathcal{H}^{(p, 0)}$ $\times \mathcal{H}^{(0, q)}$. The action by $K_{+}$obeys

$$
f(\underline{z}, \underline{w}) \in \mathcal{H}^{(p, 0)} \times \mathcal{H}^{(0, q)} \rightarrow K_{+} f(\underline{z}, \underline{w})=\underline{z} \cdot \underline{w} f(\underline{z}, \underline{w}) \in \mathcal{H}^{(p+1,0)} \times \mathcal{H}^{(0, q+1)} .
$$

On the other hand, action by $K_{-}$is the analytic equivalent of taking the trace: starting with (4.2) we get 


$$
K_{-} f(\underline{z}, \underline{w})=\sum_{p, q=0,1, \cdots}^{\infty} p q f_{j k_{1} \cdots k_{q-1}}^{j j_{1} \cdots j_{j}-1} z_{j_{1}} \cdots z_{j_{p-1}} w_{k_{1}} \cdots w_{k_{q-1}} .
$$

From these and earlier remarks we can see that the correspondences between (symmetric, traceless) tensors, entire functions, and subspaces of $\mathcal{H}$ are

$$
\begin{gathered}
\mathcal{H}^{(p, 0)} \times \mathcal{H}^{(0, q)} \leftrightarrow\left\{f_{k_{1} \cdots k_{q}}^{j_{1} \cdots j_{p}}\right\} \leftrightarrow f(\underline{z}, \underline{w}): \\
f(\lambda \underline{z}, \mu \underline{w})=\lambda^{p} \mu^{q} f(\underline{z}, \underline{w}) ; \\
f(\underline{z}, \underline{w}) \in \mathcal{H}^{(p, q ; \rho)} \Leftrightarrow f(\underline{z}, \underline{w})=(\underline{z} \cdot \underline{w})^{\rho} f_{0}(\underline{z}, \underline{w}), f_{0}(\underline{z}, \underline{w}) \in \mathcal{H}^{(p, q ; 0)} \subset \mathcal{H}^{(p, 0)} \times \mathcal{H}^{(0, q)}, \\
\frac{\partial}{\partial \underline{z}} \cdot \frac{\partial}{\partial \underline{w}} f_{0}(\underline{z}, \underline{w})=0 .
\end{gathered}
$$

Thus traceless symmetric tensors of type $(p, q)$ are in correspondence with entire functions $f_{0}(\underline{z}, \underline{w})$ of degrees of homogeneity $p$ and $q$, respectively, obeying the partial differential equation (4.9b). Alternatively, given any $f(\underline{z}, \underline{w}) \in \mathcal{H}^{(p, 0)} \times \mathcal{H}^{(0, q)}$, there is a unique "traceless" part $f_{0}(\underline{z}, \underline{w})$ belonging to the leading subspace $\mathcal{H}^{(p, q ; 0)}$ and annihilated by $K_{-}$. Thus "trace removal" can be accomplished by analytical means. We now give the procedure to pass from $f(\underline{z}, \underline{w})$ to $f_{0}(\underline{z}, \underline{w})$.

For any $f(\underline{z}, \underline{w}) \in \mathcal{H}^{(p, 0)} \times \mathcal{H}^{(0, q)}$ we can easily establish the general formula

$$
K_{-}\left\{(\underline{z} \cdot \underline{w})^{n} K_{-}^{n} f(\underline{z}, \underline{w})\right\}=n(p+q+2-n)(\underline{z} \cdot \underline{w})^{n-1} K_{-}^{n} f(\underline{z}, \underline{w})+(\underline{z} \cdot \underline{w})^{n} K_{-}^{n+1} f(\underline{z}, \underline{w}) .
$$

We try for $f_{0}(\underline{z}, \underline{w})$ the expression

$$
f_{0}(\underline{z}, \underline{w})=f(\underline{z}, \underline{w})-\sum_{n=1,2, \cdots} \alpha_{n}(\underline{z} \cdot \underline{w})^{n} K_{-}^{n} f(\underline{z}, \underline{w}),
$$

and get, using (4.10) (and omitting the arguments $\underline{z}, \underline{w}$ ),

$$
K_{-} f_{0}=K_{-} f-(p+q+1) \alpha_{1} K_{-} f-\sum_{n=1,2, \cdots}\left\{\alpha_{n}+(n+1)(p+q+1-n) \alpha_{n+1}\right\}(\underline{z} \cdot \underline{w})^{n} K_{-}^{n+1} f \text {. }
$$

We can therefore attain $K_{-} f_{0}=0$ by choosing

$$
\alpha_{n}=(-1)^{n-1} \frac{(p+q+1-n) !}{n !(p+q+1) !}, \quad n=1,2, \ldots
$$

Therefore, for any (bihomogeneous) polynomial $f(\underline{z}, \underline{w}) \in \mathcal{H}^{(p, 0)} \times \mathcal{H}^{(0, q)}$ the leading traceless part annihilated by $K_{-}$is an element $f_{0}(\underline{z}, \underline{w})$ in $\mathcal{H}^{(p, q ; 0)}$ :

$$
f_{0}(\underline{z}, \underline{w})=f(\underline{z}, \underline{w})-\sum_{n=1,2, \cdots}(-1)^{n-1} \frac{(p+q+1-n) !}{n !(p+q+1) !}(\underline{z} \cdot \underline{w})^{n} K_{-}^{n} f(\underline{z}, \underline{w}) .
$$

This result can be extended and expressed in the Fock space language. Any $|\psi\rangle \in \mathcal{H}^{(p, 0)}$ $\times \mathcal{H}^{(0, q)}$ has a unique orthogonal decomposition into various parts belonging to various UIRs of $\mathrm{SU}(3)$; using (3.25c) this reads

$$
|\psi\rangle \in \mathcal{H}^{(p, 0)} \times \mathcal{H}^{(0, q)}=\mathcal{H}^{(p, q ; 0)} \bigoplus \mathcal{H}^{(p-1, q-1 ; 1)} \bigoplus \mathcal{H}^{(p-2, q-2 ; 2)} \bigoplus \cdots:
$$




$$
\begin{gathered}
|\psi\rangle=\left|\psi_{0}\right\rangle+\left|\psi_{1}\right\rangle+\left|\psi_{2}\right\rangle+\cdots, \\
\left|\psi_{0}\right\rangle \in \mathcal{H}^{(p, q ; 0)}, K_{-}\left|\psi_{0}\right\rangle=0 ; \\
\left|\psi_{1}\right\rangle=K_{+}\left|\phi_{1}\right\rangle \in \mathcal{H}^{(p-1, q-1 ; 1)}, \\
\left|\phi_{1}\right\rangle=\frac{(p+q) !}{1 !(p+q+1) !} K_{-}\left|\psi_{1}\right\rangle \in \mathcal{H}^{(p-1, q-1 ; 0)}, \\
K_{-}^{2}\left|\psi_{1}\right\rangle=0 ; \\
\left|\psi_{2}\right\rangle=K_{+}^{2}\left|\phi_{2}\right\rangle \in \mathcal{H}^{(p-2, q-2 ; 2)}, \\
\frac{(p+q-2) !}{2 !(p+q) !} K_{-}^{2}\left|\psi_{2}\right\rangle \in \mathcal{H}^{(p-2, q-2 ; 0)}, \\
K_{-}^{3}\left|\psi_{2}\right\rangle=0 ; \cdots .
\end{gathered}
$$

The "leading" piece in $|\psi\rangle$ is thus

$$
\begin{gathered}
\left|\psi_{0}\right\rangle=|\psi\rangle-\left|\psi_{1}\right\rangle-\left|\psi_{2}\right\rangle-\cdots=|\psi\rangle-\underline{\hat{a}}^{\dagger} \cdot \underline{\hat{b}}^{\dagger}|\phi\rangle, \\
|\phi\rangle=\left|\phi_{1}\right\rangle+\underline{\hat{a}}^{\dagger} \cdot \underline{\hat{b}}^{\dagger}\left|\phi_{2}\right\rangle+\cdots \in \mathcal{H}^{(p-1,0)} \times \mathcal{H}^{(0, q-1)} .
\end{gathered}
$$

We can now infer that if to begin with we had $|\psi\rangle=\underline{\hat{a}}^{\dagger} \cdot \underline{\hat{b}}^{\dagger}|\phi\rangle$ for some $\phi \in \mathcal{H}^{(p-1,0)}$ $\times \mathcal{H}^{(0, q-1)}$, then $\left|\psi_{0}\right\rangle$ necessarily vanishes:

$$
|\psi\rangle=\underline{\hat{a}}^{\dagger} \cdot \underline{\hat{b}}^{\dagger}|\phi\rangle \Leftrightarrow\left|\psi_{0}\right\rangle=0 .
$$

In the Bargmann description this means in terms of (4.14)

$$
f(\underline{z}, \underline{w})=\underline{z} \cdot \underline{w} g(\underline{z}, \underline{w}) \Leftrightarrow f_{0}(\underline{z}, \underline{w})=0,
$$

a result which can be directly verified with some effort.

The subspace $\mathcal{H}_{0} \subset \mathcal{H}$ identified in (3.28) is describable in the Bargmann language as follows:

$$
\mathcal{H}_{0}=\left\{f(\underline{z}, \underline{w}) \in \mathcal{H} \mid \frac{\partial}{\partial \underline{z}} \cdot \frac{\partial}{\partial \underline{w}} f(\underline{z}, \underline{w})=0\right\} .
$$

In the Taylor series expansion (4.2) for such $f(\underline{z}, \underline{w})$, the tensors $f_{k_{1} \cdots k_{q}}^{j_{1} \cdots j_{p}}$ are traceless and vice versa. The squared norm and $\mathrm{SU}(3)$ action are given for $\mathcal{H}_{0}$ by (4.3) and (4.5), respectively.

\section{THE UR $\mathcal{D}_{\mathrm{SU}(2)}^{(\mathrm{IND}, 0)}$ OF SU(3)}

The Hilbert space $\mathcal{H}_{\mathrm{SU}(2)}^{(\text {ind,0) }}$ carrying the UR $\mathcal{D}_{\mathrm{SU}(2)}^{(\mathrm{ind}, 0)}$ of $\mathrm{SU}(3)$ consists of single component (scalar) complex functions on the coset space $\mathrm{SU}(3) / \mathrm{SU}(2)$. This coset space is the unit sphere in three-dimensional complex space $C^{3}$, with the natural norm and SU(3) action. Temporarily omitting the superscript zero and subscript $\mathrm{SU}(2)$ for simplicity, we have

$$
\begin{gathered}
\mathcal{H}^{(\text {ind })}=\left\{\psi(\underline{\xi}) \in C,\left.\underline{\xi} \in C^{3}\left|\|\psi\|^{2}=\int \prod_{j=1}^{3}\left(\frac{d^{2} \xi_{j}}{\pi}\right) \delta\left(\xi^{\dagger} \xi-1\right)\right| \psi(\underline{\xi})\right|^{2}\right\}, \\
\left(\mathcal{D}^{\text {(ind) }}(A) \psi\right)(\underline{\xi})=\psi\left(A^{-1} \underline{\xi}\right) .
\end{gathered}
$$


Clearly only the values of $\psi(\underline{\xi})$ for $\xi^{\dagger} \xi=1$ are relevant. For a general $\psi(\underline{\xi})$ with a Taylor series expansion we write

$$
\psi(\underline{\xi})=\sum_{p, q=0,1, \cdots}^{\infty} \psi_{k_{1} \cdots k_{q}}^{j_{1} \cdots j_{p}} \xi_{j_{1}} \cdots \xi_{j_{p}} \xi_{k_{1}}^{*} \cdots \xi_{k_{q}}^{*}
$$

[Strictly speaking, such an expansion holds only for $\psi(\underline{\xi})$ in some dense subset of $\mathcal{H}^{\text {(ind) }}$.] We note that here $\psi(\underline{\xi})$ is not an entire function of $\xi_{j}$, and since $\xi^{\dagger} \xi=1$, the tensor components $\psi_{k_{1} \cdots k_{q}}^{j_{1} \cdots j_{p}}$ may be assumed to be traceless apart from being symmetric. Then they determine $\psi(\underline{\xi})$ uniquely and vice versa.

To express the inner product $(\phi, \psi)$ for general $\phi, \psi \in \mathcal{H}^{(\text {ind) }}$ in terms of their tensor components, we need to evaluate

$$
I_{j \cdots m}^{k \cdots \cdots}=\int \prod_{j=1}^{3}\left(\frac{d^{2} \xi_{j}}{\pi}\right) \delta\left(\xi^{\dagger} \xi-1\right) \xi_{j_{1}} \cdots \xi_{j_{p}}\left(\xi_{k_{1}} \cdots \xi_{k_{q}}\right) *\left(\xi_{l_{1}} \cdots \xi_{l_{p^{\prime}}}\right) *\left(\xi_{m_{1}} \cdots \xi_{m_{q^{\prime}}}\right)
$$

for general $p, q, p^{\prime}, q^{\prime}$ and indices $j, k, l, m$. Using $\mathrm{SU}(3)$ invariance and symmetry, we see that the result must be expressible in terms of products of Kronecker deltas. Combining this with the tracelessness of the tensor components of $\phi$ and $\psi$, we can check first that we need only consider the case $p=p^{\prime}, q=q^{\prime}$, and next that

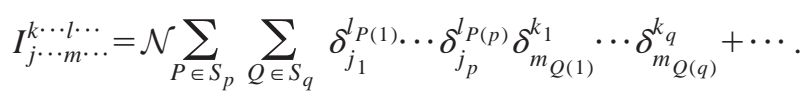

Here $\mathcal{N}$ is a normalizing factor, and the dots denote terms with factors $\delta_{j}^{k}$ or $\delta_{l}^{m}$ or both. Again the latter can be ignored. The factor $\mathcal{N}$ can be computed say by setting all $j=l=1$ and all $k=m$ $=2$ :

$$
\mathcal{N}=\frac{1}{(p+q+2) !} .
$$

We then get the result for any $\phi, \psi \in \mathcal{H}^{\text {(ind) }}$ :

$$
(\phi, \psi)=\sum_{p, q=0,1, \cdots} \frac{p ! q !}{(p+q+2) !} \phi_{k_{1} \cdots k_{q}}^{j_{1} \cdots j_{p}}{ }^{*} \psi_{k_{1} \cdots k_{q}}^{j_{1} \cdots j_{p}}
$$

With these results, all details of the induced $\mathrm{UR} \mathcal{D}_{\mathrm{SU}(2)}^{(\mathrm{ind}, 0)}$ of $\mathrm{SU}(3)$ are in hand: the Hilbert space $\mathcal{H}_{\mathrm{SU}(2)}^{(\mathrm{ind}, 0)}$ in (5.1), the expression (5.6) for inner products, and the $\mathrm{SU}(3)$ action as in (5.1).

\section{EQUIVALENCE MAP}

The full equivalence of the two UR's of SU(3), one on the subspace $\mathcal{H}_{0} \subset \mathcal{H}$ based on the six oscillator Schwinger construction of Sec. III, and the other the induced representation $\mathcal{D}_{\mathrm{SU}(2)}^{(\text {ind,0) }}$, can now be set up. The tensor component expressions (4.2) and (5.2) for vectors, and (4.3) and (5.6) for inner products, determine the one-to-one map to achieve this in full detail:

$$
\begin{gathered}
f(\underline{z}, \underline{w})=\left\{f_{k_{1} \cdots k_{q}}^{j_{1} \cdots j_{p}}\right\} \in \mathcal{H}_{0} \leftrightarrow \psi(\underline{\xi})=\left\{\psi_{k_{1} \cdots k_{q}}^{j_{1} \cdots j_{p}}\right\} \in \mathcal{H}^{(\text {ind) }}: \\
\psi_{k_{1} \cdots k_{q}}^{j_{1} \cdots j_{p}}=\sqrt{(p+q+2) !} f_{k_{1} \cdots k_{q}}^{j_{1} \cdots j_{p}}, p, q=0,1, \ldots
\end{gathered}
$$

The two inner products then match, and the $\mathrm{SU}(3)$ actions given in (4.5) and (5.1) on $f(\underline{z}, \underline{w})$ and $\psi(\underline{\xi})$ also match. 
It is worth emphasizing here the two different arguments leading to the tracelessness of the symmetric tensors on the two sides of (6.1). In the case of the left hand side, the reason is that the argument of $\psi(\underline{\xi})$ obeys the constraint $\xi^{\dagger} \xi=1$. As for the right hand side, it happens because entire functions $f(\underline{z}, \underline{w}) \in \mathcal{H}_{0}$ obey the partial differential equation in (4.19). In both cases tracelessness leads to the UR being multiplicity free, apart from being complete in the sense that all SU(3) UIR's do appear.

\section{CONCLUDING REMARKS}

To conclude, we have brought out the difficulties one encounters in naively extending the Schwinger $\mathrm{SU}(2)$ construction to $\mathrm{SU}(3)$ particularly if one wishes to retain the simplicity and economy intrinsic to the $\mathrm{SU}(2)$ case. We have shown how these difficulties can be overcome by exploiting the group $\mathrm{Sp}(2, R)$ to obtain a "generating representation" of $\mathrm{SU}(3)$ based on six bosonic oscillators. This UR of SU(3) contains all the representations of SU(3) exactly once. (It has been drawn to our attention by the referee that this result of ours is a special case of more general results available in mathematics literature. ${ }^{18,19}$ ) Further, we have shown how this "generating representation" for $\mathrm{SU}(3)$ can also be constructed using the theory of induced representations and have constructively established the equivalence between the two by making use of the Bargmann representation. It is hoped that the construction presented here will have useful applications in various branches of physics much the same way as the $\mathrm{SU}(2)$ construction has. Indeed, the work presented here has direct relevance to $\mathrm{SU}(3)$ coherent states as will be shown in a succeeding publication.

\section{ACKNOWLEDGMENTS}

This work was begun while N.M. was a Jawaharlal Nehru Chair Professor of the University of Hyderabad during September 1999. N.M. gratefully acknowledges the hospitality extended to him during his stay by the School of Physics, University of Hyderabad. We are also grateful to the referee for bringing Refs. 18 and 19 to our attention.

\section{APPENDIX: BOSON OPERATOR CONSTRUCTION OF $S U(3) \times S p(2, R)$ BASIS STATES}

We give here the explicit construction of the orthonormal basis states $|p, q ; I M Y ; m\rangle$ for $\mathcal{H}$ introduced in Eq. (3.26). We deal first with the states $|p, q ; I I Y ; k\rangle \in \mathcal{H}^{(p, q ; 0)} \subset \mathcal{H}^{(p, q)} \cap \mathcal{H}_{0}$ having highest $\mathrm{SU}(2)$ weight; then by repeated use of the $\mathrm{Sp}(2, R)$ raising operator $K_{+}=\underline{\hat{a}}^{\dagger} \cdot \underline{\hat{b}}^{\dagger}$ with $|p, q ; I I Y ; m\rangle \in \mathcal{H}^{(p, q ; m-k)} \subset \mathcal{H}^{(p, q)}$; and finally with the general state $|p, q ; I M Y ; m\rangle$ using the $\mathrm{SU}(2)$ lowering operator. At each stage the normalization will be ensured.

As is well known, the boson operators $\hat{a}_{j}^{\dagger}, \hat{b}_{j}^{\dagger}$ carry the following $\mathrm{U}(2)$ quantum numbers: ${ }^{11}$

$$
\begin{array}{cccc} 
& I & M & Y \\
\hat{a}_{1}^{\dagger}, \hat{a}_{2}^{\dagger} & \frac{1}{2} & \pm \frac{1}{2} & \frac{1}{3} \\
\hat{a}_{3}^{\dagger} & 0 & 0 & -\frac{2}{3} \\
\hat{b}_{2}^{\dagger},-\hat{b}_{1}^{\dagger} & \frac{1}{2} & \pm \frac{1}{2} & -\frac{1}{3} \\
\hat{b}_{3}^{\dagger} & 0 & 0 & \frac{2}{3}
\end{array}
$$

Therefore, $\hat{a}_{\alpha}^{\dagger} \hat{b}_{\alpha}^{\dagger} \equiv \hat{a}_{1}^{\dagger} \hat{b}_{1}^{\dagger}+\hat{a}_{2}^{\dagger} \hat{b}_{2}^{\dagger}$, $\hat{a}_{3}^{\dagger}$ and $\hat{b}_{3}^{\dagger}$ are SU(2) scalars. The I-Y multiplets present in the SU(3) UIR $(p, q)$ are listed in Eq. (2.5), and are parametrized by two integers $r, s$. The state $|p, q ; I I Y ; k\rangle \in \mathcal{H}_{0}$ involves $p$ factors $\hat{a}^{\dagger}$ and $q$ factors $\hat{b}^{\dagger}$ acting on the Fock vacuum $|\underline{0}, \underline{0}\rangle$, and in addition it is annihilated by $K_{-}=\underline{\hat{a}} \cdot \underline{\underline{b}}$. We therefore start with the expression [guided by (A1)]: 


$$
\begin{gathered}
|p, q ; I I Y ; k\rangle=\left(\hat{a}_{1}^{\dagger}\right)^{r}\left(\hat{b}_{2}^{\dagger}\right)^{s} \sum_{n=0,1, \ldots}^{(p-r, q-s)} C_{n}\left(\hat{a}_{\alpha}^{\dagger} \hat{b}_{\alpha}^{\dagger}\right)^{n}\left(\hat{a}_{3}^{\dagger}\right)^{p-r-n}\left(\hat{b}_{3}^{\dagger}\right)^{q-s-n}|\underline{0}, \underline{0}\rangle, \\
r=I+\frac{Y}{2}+\frac{1}{3}(p-q), \quad s=I-\frac{Y}{2}+\frac{1}{3}(q-p) .
\end{gathered}
$$

The condition

$$
K_{-}|p, q ; I I Y ; k\rangle=0
$$

gives the recursion relation

$$
n(r+s+n+1) C_{n}=-(p-r-n+1)(q-s-n+1) C_{n-1}, \quad n=1,2, \ldots,
$$

with the solution

$$
C_{n}=\frac{(-1)^{n}}{n !} \frac{(p-r) !(q-s) !(r+s+1) !}{(p-r-n) !(q-s-n) !(r+s+n+1) !} C_{0}, \quad n=1,2, \ldots
$$

Using this in Eq. (A2), and after some algebra, the normalized state is found to be

$$
\begin{aligned}
|p, q ; I I Y ; k\rangle= & \mathcal{N}_{p q I Y} \frac{\left(\hat{a}_{1}^{\dagger}\right)^{r}}{r !} \frac{\left(\hat{b}_{2}^{\dagger}\right)^{s}}{s !} \\
& \times \sum_{n=0,1, \ldots}^{(p-r, q-s)}<\frac{(-1)^{n}}{(r+s+n+1) !} \frac{\left(\hat{a}_{\alpha}^{\dagger} \hat{b}_{\alpha}^{\dagger}\right)^{n}}{n !} \frac{\left(\hat{a}_{3}^{\dagger}\right)^{p-r-n}}{(p-r-n) !} \frac{\left(\hat{b}_{3}^{\dagger}\right)^{q-s-n}}{(q-s-n) !}|\underline{0}, \underline{0}\rangle \in \mathcal{H}^{(p, q ; 0)}, \\
\mathcal{N}_{p q I Y}= & \{r ! s !(r+s+1) !(p-r) !(q-s) !(p+s+1) !(q+r+1) ! /(p+q+1) !\}^{1 / 2}
\end{aligned}
$$

From Eq. (3.27a) we know that vectors in $\mathcal{H}^{(p, q ; m-k)}$ for $m>k$ are obtained from vectors in $\mathcal{H}^{(p, q ; 0)}$ by applying $K_{+}^{m-k}$. Further, the normalization is controlled by Eq. (3.25b). We thus obtain

$$
|p, q ; I I Y ; m\rangle=\{(2 k-1) ! /(m-k) !(m+k-1) !\}^{1 / 2}\left(\underline{\hat{a}}^{\dagger} \cdot \underline{\hat{b}}^{\dagger}\right)^{m-k}|p, q ; I I Y ; k\rangle \in \mathcal{H}^{(p, q ; m-k)} .
$$

The last step is to reach a general value $M \leqslant I$ for the $S U(2)$ magnetic quantum number. For this we apply the $\mathrm{SU}(2)$ lowering operator $J_{-}=\hat{a}_{2}^{\dagger} \hat{a}_{1}-\hat{b}_{1}^{\dagger} \hat{b}_{2}(I-M)$ times to the state (A7), keeping track of normalization. This leads to the result

$$
|p, q ; I M Y ; m\rangle=\{(I+M) ! / 2 I !(I-M) !\}^{1 / 2}\left(\hat{a}_{2}^{\dagger} \hat{a}_{1}-\hat{b}_{1}^{\dagger} \hat{b}_{2}\right)^{I-M}|p, q ; I I Y ; m\rangle
$$

If we combine Eqs. (A6) to (A8) we get the complete expression

$$
\begin{aligned}
|p, q ; I M Y ; m\rangle= & \mathcal{N}_{p q I Y}\{(2 k-1) !(I+M) !(I-M) ! /(m-k) !(m+k-1) ! 2 I !\}^{1 / 2} \\
& \times\left(\underline{\hat{a}}^{\dagger} \cdot \underline{\hat{b}}^{\dagger}\right)^{m-k} \sum_{L=0}^{I-M} \sum_{n=0}^{(p-r, q-s)} \frac{(-1)^{n+I-M-L}}{(r+s+n+1) !} \cdot \frac{\left(\hat{a}_{\alpha}^{\dagger} \hat{b}_{\alpha}^{\dagger}\right)^{n}}{n !} \\
& \times \frac{\left(\hat{a}_{3}^{\dagger}\right)^{p-r-n}}{(p-r-n) !} \frac{\left(\hat{b}_{3}^{\dagger}\right)^{q-s-n}}{(q-s-n) !} \frac{\left(\hat{a}_{1}^{\dagger}\right)^{r-L}}{(r-L) !} \frac{\left(\hat{a}_{2}^{\dagger}\right)^{L}}{L !} \frac{\left(\hat{b}_{2}^{\dagger}\right)^{s-I+M+L}}{(s-I+M+L) !} \frac{\left(\hat{b}_{1}^{\dagger}\right)^{I-M-L}}{(I-M-L) !}|\underline{0}, \underline{0}\rangle .
\end{aligned}
$$

We thus have explicit expressions for all the normalized basis states $|p, q ; I M Y ; m\rangle$ of $\mathcal{H}$. 
${ }^{1}$ J. Schwinger, “On angular momentum," USAEC Report NYO-3071 (1952) (unpublished); reprinted in Quantum Theory of Angular Momentum, edited by L. C. Biedenharn and H. van Dam (Academic, New York, 1965); also in A Quantum Legacy-Seminal Papers of Julian Schwinger, edited by K. A. Milton (World Scientific, Singapore, 2000).

${ }^{2}$ D. P. Arovas and A. Auerbach, Phys. Rev. B 38, 316 (1988); A. Auerbach and D. P. Arovas, Phys. Rev. Lett. 61, 617 (1988); A. Auerbach, Interacting Electrons and Quantum Magnetism (Springer, New York, 1994).

${ }^{3}$ Arvind, B. Dutta, N. Mukunda, and R. Simon, Phys. Rev. A 52, 1609 (1993).

${ }^{4}$ K. Sundar, N. Mukunda, and R. Simon, J. Opt. Soc. Am. A 12, 560 (1995).

${ }^{5}$ A. P. Balachandran, P. Salomonson, B. S. Skagerstam, and J. O. Winnberg, Phys. Rev. D 15, 2308 (1977).

${ }^{6}$ M. V. Berry and J. M. Robbins, Proc. R. Soc. London, Ser. A 453, 1771 (1997).

${ }^{7}$ V. Bargmann, Rev. Mod. Phys. 34, 829 (1962).

${ }^{8}$ N. Mukunda and B. Radhakrishnan, J. Math. Phys. 15, 1320, 1332, 164, 1656 (1974).

${ }^{9}$ M. Moshinsky, Rev. Mod. Phys. 34, 813 (1962).

${ }^{10}$ G. W. Mackey, Group Representations in Hilbert Space (AMS, Providence, RI, 1963).

${ }^{11}$ See, for instance, J. J. de Swart, Rev. Mod. Phys. 35, 916 (1963).

${ }^{12}$ N. Mukunda and L. K. Pandit, J. Math. Phys. 6, 746 (1965).

${ }^{13}$ D. Sen and M. Mathur, J. Math. Phys. 42, 4181 (2001).

${ }^{14}$ N. Mukunda and L. K. Pandit, Prog. Theor. Phys. 34, 46 (1965).

${ }^{15}$ See, for instance, A. M. Perelomov, Usp. Fiz. Nauk 123, 23 (1977) [Sov. Phys. Usp. 20, 703 (1977)]; K. Wódkiewicz and J. H. Eberly, J. Opt. Soc. Am. B 2, 458 (1985).

${ }^{16}$ V. Bargmann, Ann. Math. 48, 568 (1947).

${ }^{17}$ V. Bargmann, Commun. Pure Appl. Math. 14, 187 (1961).

${ }^{18}$ R. Howe, Proc. Indian Math. Soc. 97, 85 (1987); Trans. Am. Math. Soc. 313, 539 (1989), and references cited therein.

${ }^{19}$ I. M. Gelfand and A. V. Zelevinskii, Funct. Anal. Appl. 18, 183 (1984). 